\title{
Presentación oral
}

\section{Prevención de eventos cardiovasculares en la población colombiana con diabetes mellitus tipo 2 (DM2) durante el último trimestre de 2008 y 2018}

\author{
Seudónimo: Saturno
}

Antecedentes: la DM2 es una patología que aumenta el riesgo cardiovascular. No hay estudios en Colombia que evalúen la prevención cardiovascular a lo largo del tiempo.

Materiales y métodos: estudio descriptivo transversal que incluyó pacientes con DM2 cuya medicación fuera dispensada por Audifarma S. A. durante el último trimestre de 2008 y 2018. Se aplicó una estadística descriptiva, pruebas $\mathrm{X}^{2}$ para la comparación de variables categóricas y un modelo de regresión logística binaria para el ajuste de las variables asociadas $(p<0,05)$ con prescripción de aspirina y estatinas. Los análisis fueron realizados con SPSS v.24.0.

Resultados: la población total de pacientes con DM2 en tratamiento fue de 26741 pacientes en 2008 y 188321 pacientes en 2018. La estatina con mayor prescripción para 2008 fue lovastatina $(98,7 \%)$ y para atorvastatina $(85,3 \%)$ para 2018; el uso de estatinas de alta intensidad durante el último trimestre del 2008 y 2018 fue de 1,1 \% y 95,2 \%, respectivamente. La probabilidad del uso de aspirina y estatinas en 2008 y 2018 se asoció significativamente con la progresión de la edad ( $\geq 75$ años, Odds ratio [OR]: 6,488 y 5,819 ) y clopidogrel (OR: 5,854 y 2,238), y disminuyó con uso de anticoagulantes directos para 2018 (OR: 0,149).

Conclusión: en pacientes con DM2 se evidenció un aumento en la prescripción de estatinas de alta intensidad, un dato relevante debido al impacto en los desenlaces de eventos cardiovasculares. Se encontró una probabilidad mayor de prescripción de aspirina y estatinas con la progresión de la edad y la comedicación con clopidogrel.

Palabras clave: diabetes mellitus tipo 2, enfermedades cardiovasculares, prevención \& control, aspirina, inhibidores de hidroximetilglutaril-CoA Reductasas (DeCS).

\section{METABOLISMO ÓSEO}

\section{Presentación oral}

\section{Niveles de 25-hidroxivitamina $\mathrm{D}$ en una población estudiantil universitaria de Armenia, Colombia}

Seudónimo: LJPV, DCG, OANC

Objetivo: describir la prevalencia de la deficiencia e insuficiencia de 25-hidroxivitamina D en universitarios de Quindío, Colombia.

Materiales y métodos: se realizó un estudio descriptivo de corte transversal durante 2017-2018. Se realizó una encuesta epidemiológica y se tomaron medidas antropométricas y una muestra de sangre de la que se midieron los ni- veles de 25-hidroxivitamina D mediante la técnica de ELFA (Enzyme Linked Fluorescent Assay). Se realizaron análisis de la varianza ANOVA (ANalysis Of VAriance) para las variables cuantitativas y la prueba de Chi-Cuadrado para las variables categóricas. Se consideró un nivel de significancia cuando el valor fue $p<0,05$. 
Resultados: se seleccionaron 153 individuos. La edad promedio fue 21,05 años y el promedio de vitamina D fue $27,7 \mathrm{ng} /$ $\mathrm{mL}$. Se encontraron diferencias estadísticamente significativas por sexo $(p=0,009)$ en los niveles de vitamina D. La insuficiencia (20-30 ng/mL) se presentó en $39,22 \%$ en las mujeres y $18,95 \%$ en los hombres. El porcentaje de deficiencia fue mayor en las mujeres $(7,19 \%)$ que en los hombres $(3,27 \%)$.

Conclusiones: se obtuvo el primer registro de la prevalencia de vitamina D en esta población. La deficiencia e insuficiencia de vitamina $\mathrm{D}$ fueron condiciones prevalentes en la población estudiada, con frecuencias del 10,46 \% y 58,17 \%, respectivamente; en ambos casos fue mayor en el sexo femenino.

Palabras clave: vitamina D, calcitriol, deficiencia, factores de riesgo.

\section{Abstract}

Objective: to describe the prevalence of 25-hydroxyvitamin D deficiency and insufficiency among university students of Quindío, east of Colombia.

Materials and methods: a descriptive cross-sectional study was carried out during 2017-2018. An epidemiological survey was conducted, anthropometric measures were taken, and blood samples collected, from which the levels of 25-hydroxyvitamin D were measured by means of ELFA assay. Analysis by ANOVA was performed for the quantitative variables, and Chi-square test for categorical variables. Significance was established as $p<0.05$.

Results: a total of one hundred and fifty-three individuals were included in the study. The average age was 21.05 years, and the vitamin D median concentration was $27.7 \mathrm{ng} / \mathrm{mL}$. Statistically significant differences were found after adjusting for sex ( $p=0.009$ ). 25- hydroxyvitamin D insufficiency (defined as concentrations ranging between $20-30 \mathrm{ng} / \mathrm{mL}$ ) was found in $39.22 \%$ of female and $18.95 \%$ of male participants. The deficiency, in turn, was found to be higher in females $(7.19 \%)$ than in males (3.27\%).

Conclusions: this study constitutes the first report of the prevalence of 25-hydroxyvitamin D insufficiency and deficiency in this Colombian population. Both deficiency and insufficiency were prevalent conditions in the study population, with total frequencies of $10.46 \%$ and $58.17 \%$ respectively; women turned out to be more frequently affected by these.

Key words: Vitamin D, Calcitriol, Deficiency, Risk Factors.

\section{Presentación oral}

\section{Niveles de vitamina D séricos y en lavado broncoalveolar en pacientes con sospecha de tuberculosis pulmonar}

\section{Seudónimo: Vitaminadbal}

Introducción: la tuberculosis es una enfermedad de alta prevalencia a nivel mundial, con una importante carga de morbimortalidad. Múltiples factores contribuyen a la presentación de esta enfermedad, incluyendo el seroestado de vitamina D. Se pretende medir el nivel local (broncoalveolar) y sistémico del compuesto en pacientes con tuberculosis y controles sin la enfermedad.

Metodología: se realizó un estudio en dos fases. La primera fase con muestras de pacientes de un trabajo local previo; la segunda fase prospectiva se realizó entre enero de 2018-diciembre de 2018, con pacientes a los que se les indicó la realización de una fibrobroncoscopia debido a la sospecha de tuberculosis u otras causas. Se realizó una medición de vitamina
D en suero, en el lavado broncoalveolar y estudios adicionales de metabolismo de calcio. Los datos se procesaron con el programa SPSS versión 23 y se aplicaron estadísticas descriptivas, medidas de tendencia central, dispersión y posición. Se empleó la prueba de Kuskal Wallis para evaluar la asociación entre las variables consideradas, con un nivel de significancia de un valor de $p<0,05$.

Resultados: ingresaron 52 pacientes: 18 se clasificaron como pacientes con tuberculosis, 24 como pacientes con controles infecciosos y 10 como pacientes con controles no infecciosos. Las características de la población en los tres grupos fueron homogéneas. Los niveles séricos de vitamina D de los pacientes con tuberculosis fueron más bajos que en los otros 
dos grupos, con una media de $22,4 \mathrm{ng} / \mathrm{mL}$ frente a $33 \mathrm{ng} / \mathrm{mL}$ (controles infecciosos) y $32,6 \mathrm{ng} / \mathrm{mL}$ (controles no infecciosos) $(p=0,006)$. En el lavado broncoalveolar, los niveles de vitamina D fueron más bajos en los pacientes con tuberculosis, con media de 9,7 ng/mL en comparación con los controles infecciosos y los no infecciosos $(p=0,012)$. No hubo diferencias en los niveles de calcio y albúmina en los tres grupos.

Conclusiones: hubo una diferencia significativamente estadística entre los niveles séricos de vitamina D y el lavado broncoalveolar de los pacientes con tuberculosis pulmonar, que guardó relación con el comportamiento local de esta. Se debe evaluar el papel de la vitamina $\mathrm{D}$ tanto a nivel diagnóstico, como a nivel potencialmente terapéutico en pacientes con tuberculosis pulmonar.

Palabras clave: tuberculosis, vitamina D, lavado broncoalveolar. 